\title{
Static Anti-windup Design for a Class of Nonlinear Systems
}

\author{
J. M. Gomes da Silva Jr. ${ }^{1 *}$, M. Z. Oliveira ${ }^{1,2}$, D. Coutinho ${ }^{3}$ and S. Tarbouriech ${ }^{4}$
}

1. UFRGS - Department of Electrical Engineering,

Av. Osvaldo Aranha 103, 90035-190 Porto Alegre-RS, Brazil.

e-mail: jmgomes@ece.ufrgs.br

* Corresponding Author

2. University of Caxias do Sul - UCS,

Rua Francisco Getulio Vargas 1131, 95050-560, Caxias do Sul-RS, Brazil

e-mail: mauricio.zardo@ucs.br

3. UFSC - Department of Automation and Systems,

PO Box 476, 88040-900, Florianópolis, Brazil

e-mail: daniel.coutinho@ufsc.br

4. CNRS, LAAS, 7 avenue du colonel Roche, F-31400 Toulouse, France and Univ de Toulouse, LAAS, F-31400 Toulouse, France.

e-mail: tarbour@laas.fr

\begin{abstract}
This paper focuses on the problem of static anti-windup design for a class of multivariable nonlinear systems subject to actuator saturation. The considered class regards all systems that are rational on the states or that can be conveniently represented by a rational system with algebraic constraints considering some variable changes. More precisely, a method is proposed to compute a static anti-windup gain which ensures regional stability for the closed-loop system assuming that a dynamic output feedback controller is previously designed to stabilize the nonlinear system. The results are based on a differential-algebraic representation of rational systems. The control saturation effects are taken into account by the application of a generalized sector bound condition. From these elements, LMI-based conditions are devised to compute an anti-windup gain with the aim of enlarging the closed-loop region of attraction. Several numerical examples are provided to illustrate the application of the proposed method.
\end{abstract}




\section{Introduction}

The general principle of the anti-windup technique is the introduction of an extra feedback loop in a predesigned control system to mitigate the effects caused by saturation. Although there exist many different strategies and design approaches, the majority of the results regards the synthesis of anti-windup compensators for linear systems (see [1-6] and references therein). However, if the anti-windup compensator is designed based on the linear approximation of the nonlinear dynamics, it can lead to a poor behavior when implemented on the original nonlinear control system. Moreover, the computed region of attraction of the closed-loop system considering the linear approximation may be highly modified by the nonlinear dynamics. In general, one cannot ensure that a region of stability computed considering the linear approximation will be valid for the actual nonlinear system. In spite of these facts, only few works have addressed the antiwindup synthesis problem for nonlinear systems subject to saturating actuators. We can cite, for instance, the references [7-9] which consider anti-windup synthesis for linear-parameter varying systems, [10] which proposes anti-windup methods for Euler-Lagrange systems, and [11] which considers an adaptive control design. We can also cite some works dealing with the anti-windup synthesis for systems with nonlinear dynamic inversion (NDI) controllers, such as the references [12-16].

On the other hand, a key problem to characterize the stability of nonlinear systems is the determination of a non conservative estimate of the system region of attraction. In general, the estimates are obtained from Lyapunov level sets (see, for instance, the references [17-21]). In this context, considering an NDI-based controller, the synthesis of a dynamic anti-windup compensator aiming at the enlargement of an estimate of the region of attraction of the closed-loop system in the subspace of the plant variables has been proposed in [22] for the class of quadratic systems. The considered architecture in this case, as in [12], can be seen as a generalization of the coprime-factorization approach (or the model recovery anti-windup) used in linear anti-windup [2,23]. This same problem has been tackled in [24], but considering rational systems and static anti-windup compensation. In particular, the multivariable case is not addressed and the method is based on a non-convex condition, although the problem solution is obtained from LMI relaxations. These drawbacks have been in part overcome in [25], where convex stabilizing conditions are proposed. However, it should be pointed out that in [24] and [25], only systems and controllers with linear outputs can be considered. It turns out that these approaches cannot be applied in the case of NDI controllers, for instance.

In light of the above scenario, this paper aims at devising a numerical and tractable technique to design static anti-windup compensators for a class of nonlinear systems subject to actuator saturation. The class of systems considered in this paper covers all systems modeled by rational differential equations. The motivation to investigate rational systems is their use to model several phenomena in real life applications, in particular in systems biology, engineering, physics, and economics, and also in nonlinear system identification and realization theory $[26,27]$, which has given rise to a large number of works on the stability analysis, control and filter design for rational nonlinear systems [19,28-35]. We emphasize that a large class of systems can 
be embedded in this setup such as quadratic and polynomial systems. Furthermore, the proposed technique can deal with more complex nonlinearities by means of additional algebraic constraints and/or change of variables (see, e.g., [32-34]). In particular, the method to be presented in the paper applies a differential algebraic representation (DAR) of nonlinear systems letting to cast Lyapunov based stability conditions in terms of a finite set of state-dependent linear matrix inequalities, which can be numerically solved at the vertices of a given polytope of admissible states. To deal with the saturation nonlinearity, a modified version of the generalized sector bound condition proposed in [6] is also considered. From these elements, we derive regional stabilizing conditions directly in LMI form. In addition, an LMI-based optimization problem is devised to compute an anti-windup gain in order to obtain a maximized region of asymptotic stability, which implicitly leads to the maximization of the basin of attraction of the closed-loop system. This work is a further development of our previous result proposed in [24], where the main differences and advantages are: (a) the conditions are directly cast in terms of LMIs avoiding iterative relaxation schemes, and (b) it allows to consider multivariable nonlinear control systems in a straightforward way. The paper can also be seen as a generalization of our recent conference work [25], in the sense that systems and controllers with rational outputs can now be treated, which allows to cope with the important case of NDI controllers, among others.

The paper is organized as follows. Section 2 introduces the problem to be addressed in the paper. Section 3 provides preliminary results concerning the system representation, the Lyapunov theory, and the generalized sector bound condition. The main result is presented in Section 4, where the computation of the anti-windup gain is obtained by means of an optimization problem in Section 4.1. Several illustrative examples are provided in Section 5 in order to demonstrate the potentialities of the proposed approach. Section 6 ends the paper with some concluding remarks.

Notation: $I_{n}$ is the $n \times n$ identity matrix and 0 may either denote the scalar zero or a matrix of zeros with appropriate dimensions. For a real matrix $H, H^{\prime}$ denotes its transpose and $H>0$ means that $H$ is symmetric and positive definite. $H_{(i)}$ and $x_{(i)}$ denotes the $i^{\text {th }}$ line of matrix $H$ and the $i^{\text {th }}$ element of vector $x$, respectively. For a block matrix, the symbol $\star$ represents symmetric blocks outside the main diagonal block. For a given polytope $\Phi, \mathcal{V}(\Phi)$ is the set of vertices of $\Phi$. blockdiag $(\cdots)$ is a block diagonal matrix whose diagonal blocks are the ordered arguments. Matrix and vector dimensions are omitted whenever they can be inferred from the context.

\section{Problem Statement}

Consider the following class of nonlinear control systems:

$$
\begin{aligned}
\dot{x}(t) & =f_{x}(x(t))+g(x(t)) \operatorname{sat}\left(v_{c}(t)\right) \\
y(t) & =h_{y}(x(t))
\end{aligned}
$$


where $x \in \mathcal{B}_{x} \subset \mathbb{R}^{n}$ denotes the state vector; $y \in \mathbb{R}^{n_{y}}$ is the measured output; $v_{c} \in \mathbb{R}^{n_{v}}$ is the control input; $\operatorname{sat}(\cdot)$ is a classical saturation function, defined as follows:

$$
\operatorname{sat}\left(v_{c(i)}(t)\right)=\operatorname{sign}\left(v_{c(i)}(t)\right) \min \left\{\left|v_{c(i)}(t)\right|, u_{0(i)}\right\}, i=1, \ldots, n_{v}
$$

with $u_{0(i)}>0, i=1, \ldots, n_{v}$ denoting the symmetric saturation level of the $i^{t h}$ actuator. It is assumed that $f_{x}: \mathbb{R}^{n} \mapsto \mathbb{R}^{n}, g: \mathbb{R}^{n} \mapsto \mathbb{R}^{n} \times \mathbb{R}^{n_{v}}, h_{y}: \mathbb{R}^{n} \mapsto \mathbb{R}^{n_{y}}$ are rational functions of $x$ satisfying the conditions for the existence and uniqueness of solution for all $x \in \mathcal{B}_{x}$.

In addition, we assume that a dynamic output stabilizing controller

$$
\begin{aligned}
\dot{\eta}(t) & =f_{\eta}(\eta(t), y(t)) \\
v_{c}(t) & =h_{\eta}(\eta(t), y(t))
\end{aligned}
$$

has been designed to guarantee some performance requirements (such as exponential decay rate and damping of the state time-response) and the stability of the closed-loop system (1)-(2) in the absence of control saturation, where: $\eta \in \mathcal{B}_{\eta} \subset \mathbb{R}^{n_{c}}$ denotes the controller state; $y(t)$ is the controller input; $v_{c}(t)$ is the controller output; $f_{\eta}: \mathbb{R}^{n_{c}} \times \mathbb{R}^{n_{y}} \mapsto \mathbb{R}^{n_{c}}$ and $h_{\eta}: \mathbb{R}^{n_{c}} \times \mathbb{R}^{n_{y}} \mapsto \mathbb{R}^{n_{v}}$ are rational functions of $\eta$ and $y$ satisfying the conditions for existence and solutions for all $\eta \in \mathcal{B}_{\eta}$ and $x \in \mathcal{B}_{x}$.

In view of the undesirable effects of windup caused by input saturation, an anti-windup gain is added to the controller. Thus, considering the dynamic controller and the anti-windup strategy, the closed-loop system reads:

$$
\begin{aligned}
\dot{x}(t) & =f_{x}(x(t))+g(x(t)) \operatorname{sat}\left(v_{c}(t)\right) \\
y(t) & =h_{y}(x(t)) \\
\dot{\eta}(t) & =f_{\eta}(\eta(t), y(t))+E_{c}\left(\operatorname{sat}\left(v_{c}(t)\right)-v_{c}(t)\right) \\
v_{c}(t) & =h_{\eta}(\eta(t), y(t))
\end{aligned}
$$

where $E_{c} \in \mathbb{R}^{n_{c} \times n_{v}}$ is a constant matrix representing the anti-windup gain to be determined.

Considering the above setup, we aim at determining the anti-windup gain $E_{c}$ such that the region of attraction of the closed-loop system (3) is enlarged.

\section{Preliminaries}

This section presents some basic results needed to derive an LMI-based method to address the anti-windup computation as stated in Section 2. Firstly, we present the Differential Algebraic Representation (DAR) of nonlinear systems. Then we briefly recall some results of the Lyapunov Theory. Finally, we present an extension of the generalized sector condition proposed in [6] to cope with the case where the actuator input is computed as a rational function of both the plant and controller states. This condition will be useful for dealing with the saturation nonlinearity in the sequel. 


\subsection{Differential Algebraic Representation - DAR}

Firstly, define a decentralized deadzone nonlinearity

$$
\psi\left(v_{c}(t)\right) \triangleq v_{c}(t)-\operatorname{sat}\left(v_{c}(t)\right)
$$

and rewrite system (3) as follows:

$$
\begin{aligned}
\dot{x}(t) & =f_{x}(x(t))+g(x(t)) v_{c}(t)-g(x(t)) \psi\left(v_{c}(t)\right) \\
y(t) & =h_{y}(x(t)) \\
\dot{\eta}(t) & =f_{\eta}(\eta(t), y(t))-E_{c} \psi\left(v_{c}(t)\right) \\
v_{c}(t) & =h_{\eta}(\eta(t), y(t)) .
\end{aligned}
$$

It is worth noticing that, since $h_{y}$ and $h_{\eta}$ are rational functions, the resulting closed-loop system (5) is indeed rational in the state variables $x$ and $\eta$. Hence, defining an augmented state vector $\xi(t)=\left[x(t)^{\prime} \eta(t)^{\prime}\right]^{\prime} \in$ $\mathcal{B}_{\xi} \subset \mathbb{R}^{n_{\xi}}, \mathcal{B}_{\xi}=\left\{\xi \in \mathbb{R}^{n_{\xi}} ; x \in \mathcal{B}_{x}\right.$ and $\left.\eta \in \mathcal{B}_{\eta}\right\}$, with $n_{\xi}=n+n_{c}$, it is always possible to find a Differential Algebraic Representation (DAR) for (5), as follows:

$$
\begin{aligned}
\dot{\xi}(t) & =\mathcal{A}_{a} \xi(t)+\mathcal{A}_{b} z(t)+\left(\mathcal{A}_{c}-\mathcal{W} E_{c}\right) \psi\left(v_{c}(t)\right) \\
0 & =\Omega_{a} \xi(t)+\Omega_{b} z(t)+\Omega_{c} \psi\left(v_{c}(t)\right)
\end{aligned}
$$

where $\mathcal{W}=\left[\begin{array}{c}0_{n \times n_{c}} \\ I_{n_{c}}\end{array}\right] ; z \in \mathbb{R}^{n_{z}}$ is an auxiliary nonlinear vector function with respect to (w.r.t.) $\xi$ and linear w.r.t. $\psi$, containing rational and polynomial terms (having terms of order equal or larger than two); $\mathcal{A}_{a} \in \mathbb{R}^{n_{\xi} \times n_{\xi}}, \mathcal{A}_{b} \in \mathbb{R}^{n_{\xi} \times n_{z}}, \mathcal{A}_{c} \in \mathbb{R}^{n_{\xi} \times n_{v}}, \Omega_{a} \in \mathbb{R}^{n_{z} \times n_{\xi}}, \Omega_{b} \in \mathbb{R}^{n_{z} \times n_{z}}$ and $\Omega_{c} \in \mathbb{R}^{n_{z} \times n_{v}}$, are affine matrix functions of $\xi$.

Similarly, it is always possible to re-write $v_{c}(t)$ in the following form since $h_{\eta}$ is a rational function of $\xi$ :

$$
\begin{gathered}
v_{c}(t)=K_{1} \xi(t)+K_{2} \rho(t) \\
0=\Xi_{1} \xi(t)+\Xi_{2} \rho(t)
\end{gathered}
$$

where $\rho \in \mathbb{R}^{n_{\rho}}$ is an auxiliary nonlinear vector function of $(x, \eta)$ containing rational and polynomial terms (having terms of order equal or larger than two) of $h_{\eta}$, and $K_{1} \in \mathbb{R}^{n_{v} \times n_{\xi}}, K_{2} \in \mathbb{R}^{n_{v} \times n_{\rho}}, \Xi_{1} \in \mathbb{R}^{n_{\rho} \times n_{\xi}}$ and $\Xi_{2} \in \mathbb{R}^{n_{\rho} \times n_{\rho}}$ are affine matrix functions of $\xi$.

Regarding the closed-loop system (5) (or (6)-(7)), we assume that:

(A1) the origin $(\xi=0)$ is a (locally) asymptotically stable equilibrium point; and

(A2) the domain $\mathcal{B}_{\xi}$ is a given polytope containing the origin in its interior.

Hence, the polytope $\mathcal{B}_{\xi}$ can be described by a set of scalar inequalities as follows:

$$
\mathcal{B}_{\xi}=\left\{\xi \in \mathbb{R}^{n_{\xi}}: q_{r}^{\prime} \xi \leq 1, r=1, \ldots, n_{e}\right\}
$$

where $q_{r} \in \mathbb{R}^{n_{\xi}}, r=1, \ldots, n_{e}$, are given vectors defining the $n_{e}$ faces of $\mathcal{B}_{\xi}$. For convenience, $\mathcal{B}_{\xi}$ can be alternatively described by the convex hull of its vertices, where the notation $\mathcal{V}\left(\mathcal{B}_{\xi}\right)$ denotes the set of vertices 
of $\mathcal{B}_{\xi}$. Indeed, suppose that $\mathcal{B}_{\xi}$ has $N_{v}$ vertices, i.e. $\mathcal{V}\left(\mathcal{B}_{\xi}\right)=\left\{v_{1}, v_{2}, \ldots, v_{N_{v}}\right\}$, it follows that any $\xi \in \mathcal{B}_{\xi}$ can be computed as a convex combination of these vertices, that is:

$$
\forall \xi \in \mathcal{B}_{\xi}, \quad \xi=\sum_{i=1}^{N_{v}} \lambda_{i} v_{i}
$$

with $\lambda_{i} \geq 0$ and $\sum_{i=1}^{N_{v}} \lambda_{i}=1$.

To guarantee that the DAR (6)-(7) is well posed (i.e., the uniqueness of the solution $\xi(t)$ is ensured), we further consider that:

(A3) the matrix functions $\Omega_{b}$ and $\Xi_{2}$, affine on $\xi$, have full rank for all $\xi \in \mathcal{B}_{\xi}$.

Notice from A3 that the auxiliary vectors $z(t)$ and $\rho(t)$ can be eliminated, respectively, from (6) and (7) leading to the original system representation in (5) by means of

$$
z(t)=-\Omega_{b}^{-1}\left(\Omega_{a} \xi(t)+\Omega_{c} \psi\left(v_{c}(t)\right)\right) \text { and } \rho(t)=-\Xi_{2}^{-1} \Xi_{1} \xi(t)
$$

It turns out that a broad class of nonlinear systems can be embedded in the DAR as defined in (6) and (7), such as the whole class of (nonsingular) rational control systems which are linear w.r.t. the control input $u=\operatorname{sat}\left(v_{c}(t)\right)$. Moreover, more complex nonlinearities can be dealt with by applying some change of variables or by adding algebraic conditions. See, for instance, some examples in references [19, 32-34]. Moreover, it has has been shown in [34] that the DAR includes the linear fractional representation (LFR) of nonlinear systems introduced in [28] and thus we may apply the LFR modeling tools (see, e.g., [29]) to derive a DAR model for (nonsingular) rational control systems.

Remark 1 Although the DAR representation presents some similarity to quasi-LPV models (see, for instance, [36] and references therein) some key differences should be highlighted. In the quasi-LPV approach the nonlinearities are seen as bounded time-varying parameters. However, once the representation is obtained, these parameters are treated as completely independent of the state dynamics, which is potentially conservative for assessing the stability of nonlinear systems. On the other hand, in the DAR approach, the variables $z$ (and $\rho$ ), which concentrate the nonlinearities, are directly coupled with the true state through an algebraic equation. Moreover, the set $\mathcal{B}_{\xi}$, is considered directly to check stability by convexity arguments, while in the LPV approach, this set should be re-defined according to the definition of the varying parameter. In order to illustrate these differences, consider the following simple polynomial system:

$$
\dot{\xi}(t)=\left(\xi(t)^{2}-10\right) \xi(t), \xi(t) \in \mathcal{B}_{\xi}:=\{\xi:|\xi| \leq \alpha\}
$$

If we consider the quasi-LPV framework, the above system may be represented in terms of a parameter varying function

$$
\theta(t)=\xi(t)^{2}, \theta(t) \in \mathcal{B}_{\theta}:=\left\{\theta: 0 \leq \theta \leq \alpha^{2}\right\}
$$

yielding the following representation for the system in (10)

$$
\dot{\xi}(t)=(\theta(t)-10) \xi(t), \theta(t) \in \mathcal{B}_{\theta} .
$$


On the other hand, a DAR for the system in (10) can be obtained by defining $z(t)=\xi(t)^{2}$ leading to the following representation:

$$
\left\{\begin{aligned}
\dot{\xi}(t) & =-10 \xi(t)+z(t) \quad, \xi(t) \in \mathcal{B}_{\xi} \\
0 & =\Omega_{a} \xi(t)+\Omega_{b} z(t)
\end{aligned}\right.
$$

where $\Omega_{a}=\xi$ and $\Omega_{b}=-1$. Notice, for large values of $\alpha$, that the representation in (11) is likely to be more conservative than the DAR in (12).

\subsection{Lyapunov Theory Results}

In this section, we recall a basic result from the Lyapunov theory [17].

Lemma 1 Consider a nonlinear system $\dot{\xi}=a(\xi)$ where $a: \mathcal{B}_{\xi} \mapsto \mathcal{B}_{\xi}, \mathcal{B}_{\xi} \subset \mathbb{R}^{n_{\xi}}$, is a locally Lipschitz function such that $a(0)=0$. Suppose there exist positive scalars $\epsilon_{1}, \epsilon_{2}$ and $\epsilon_{3}$, and a continuously differentiable function $V: \mathcal{B}_{\xi} \mapsto \mathbb{R}$ satisfying the following conditions:

$$
\begin{gathered}
\epsilon_{1} \xi^{\prime} \xi \leq V(\xi) \leq \epsilon_{2} \xi^{\prime} \xi, \forall \xi \in \mathcal{B}_{\xi}, \\
\dot{V}(\xi) \leq-\epsilon_{3} \xi^{\prime} \xi, \forall \xi \in \mathcal{B}_{\xi}, \\
\mathcal{R} \triangleq\left\{\xi \in \mathbb{R}^{n_{\xi}}: V(\xi) \leq c\right\} \subset \mathcal{B}_{\xi},
\end{gathered}
$$

then, $V(\xi)$ is a Lyapunov function in $\mathcal{B}_{\xi}$. Moreover, for all $\xi(0) \in \mathcal{R}$ the trajectory $\xi(t)$ belongs to $\mathcal{R}$ and approaches the origin as $t \rightarrow \infty$.

To assess the local stability of system (6), in this work we consider a quadratic Lyapunov function:

$$
V(\xi)=\xi^{\prime} P \xi, P=P^{\prime}>0
$$

with $P \in \mathbb{R}^{n_{\xi} \times n_{\xi}}$, and the following normalized level set

$$
\mathcal{R}=\left\{\xi \in \mathbb{R}^{n}: \xi^{\prime} P \xi \leq 1\right\}
$$

In view of Lemma 1 , if $V(\xi)$ as above defined satisfies the conditions (13)-(15), for all $x \in \mathcal{B}_{\xi}$ and $\mathcal{R} \subset \mathcal{B}_{\xi}$, then $\mathcal{R}$ is an invariant and contractive set contained in the region of attraction of the nonlinear system and can be seen as an estimate of it [17].

\subsection{Generalized Sector Bound Condition}

Let $G_{1} \in \mathbb{R}^{n_{v} \times n_{\xi}}$ and $G_{2} \in \mathbb{R}^{n_{v} \times n_{\rho}}$ be affine matrix functions of $\xi$. Define now the following set

$$
\mathcal{S} \triangleq\left\{\xi \in \mathbb{R}^{n_{\xi}}:\left|\left(K_{1(i)}-G_{1(i)}\right) \xi+\left(K_{2(i)}-G_{2(i)}\right) \rho\right| \leq u_{0(i)}, \quad i=1, \ldots, n_{v}\right\}
$$

Note that $\rho$ is a function of $\xi$ and thus $\mathcal{S}$ defines a set of vectors in the $\xi$ domain.

From the deadzone nonlinearity $\psi\left(v_{c}\right)$ defined in $(4)$ and the set $\mathcal{S}$ as above defined, the following Lemma can be stated as a generalization of the Lemma 1 presented in [6] (see also [37]). 
Lemma 2 If $\xi \in \mathcal{S}$ then the relation

$$
\psi\left(v_{c}\right)^{\prime} T\left[\psi\left(v_{c}\right)-G_{1} \xi-G_{2} \rho\right] \leq 0
$$

is verified for any diagonal and positive definite matrix $T \in \mathbb{R}^{n_{v} \times n_{v}}$.

Proof. Recall that $v_{c}=K_{1} \xi+K_{2} \rho$ and define $r=G_{1} \xi+G_{2} \rho$. Considering $\xi \in \mathcal{S}$, it follows that $\left(v_{c(i)}-r_{(i)}-\right.$ $\left.u_{0(i)}\right)<0$ and $\left(v_{c(i)}-r_{(i)}+u_{0(i)}\right)>0, i=1 \ldots, n_{v}$. If $v_{c(i)}>u_{0(i)}$, it follows that $\psi\left(v_{c}\right)_{(i)}=v_{c(i)}-u_{0(i)}>0$ and then $\psi\left(v_{c}\right)_{(i)}\left(v_{c(i)}-r_{(i)}-u_{0(i)}\right)<0$. If $v_{c(i)}<-u_{0(i)}$, it follows that $\psi\left(v_{c}\right)_{(i)}=v_{c(i)}+u_{0(i)}<0$ and then $\psi\left(v_{c}\right)_{(i)}\left(v_{c(i)}-r_{(i)}+u_{0(i)}\right)<0$. Finally, if $-u_{0(i)} \leq v_{c(i)} \leq u_{0(i)}$, it follows that $\psi\left(v_{c}\right)_{(i)}=0$. From all this cases, we can conclude that $(19)$ is verified for any diagonal and positive definite matrix $T \in \mathbb{R}^{n_{v} \times n_{v}}$.

For deadzone nonlinearities, the relation (19) can be viewed as a generalized sector condition which encompasses the classical one used, for instance, in [38] and [39]. The generalized sector condition is known to be less conservative than the classical one when assessing the stability of systems subject to actuator saturation [6]. Furthermore, it allows to convexify the problem of synthesizing local stabilizing anti-windup compensators (see [37]). In the present form, it should be noticed that matrices $G_{1}$ and $G_{2}$ can be affine in $\xi$. As it will be seen further, these matrices will be considered as free variables in the synthesis optimization problem.

\section{Main Result}

In this section, an LMI framework to address the anti-windup synthesis problem stated in Section 2 is presented.

In this case, by considering the quadratic Lyapunov function defined in (16), it follows that:

$$
\dot{V}(\xi)=\dot{\xi}(t)^{\prime} P \xi(t)+\xi(t)^{\prime} P \dot{\xi}(t)
$$

Considering the auxiliary vector $\zeta_{0}=\left[\begin{array}{ll}\dot{\xi}(t)^{\prime} & \xi(t)^{\prime}\end{array}\right]^{\prime}$, we can rewrite $(20)$ as $\dot{V}(\xi)=\zeta_{0}^{\prime} \Lambda_{1} \zeta_{0}$ with $\Lambda_{1}=\left[\begin{array}{ll}0 & P \\ P & 0\end{array}\right]$.

In view of Lemma 2 , if $\xi \in \mathcal{S}$, then the relation $\psi\left(v_{c}\right)^{\prime} T\left[\psi\left(v_{c}\right)-G_{1} \xi-G_{2} \rho\right] \leq 0$ is verified for any matrix $T$ diagonal and positive definite. Hence, if

$$
\zeta_{0}^{\prime} \Lambda_{1} \zeta_{0}-2 \psi\left(v_{c}\right)^{\prime} T\left[\psi\left(v_{c}\right)-G_{1} \xi-G_{2} \rho\right]<0, \forall \xi \in \mathcal{B}_{\xi} \cap \mathcal{S}
$$

is verified, then $\dot{V}(\xi)<0$ for all $\xi \in \mathcal{S} \cap \mathcal{B}_{\xi}$.

Considering the auxiliary vector $\zeta=\left[\begin{array}{lllll}\dot{\xi}(t)^{\prime} & \xi(t)^{\prime} & z(t)^{\prime} & \rho(t)^{\prime} & \psi\left(v_{c}(t)\right)^{\prime}\end{array}\right]^{\prime} \quad$ we can rewrite $(21)$ as

$$
\zeta^{\prime} \Lambda_{2} \zeta<0
$$


with

Define now the following scalars:

$$
\Lambda_{2}=\left[\begin{array}{ccccc}
0 & P & 0 & 0 & 0 \\
P & 0 & 0 & 0 & G_{1}^{\prime} T \\
0 & 0 & 0 & 0 & 0 \\
0 & 0 & 0 & 0 & G_{2}^{\prime} T \\
0 & T G_{1} & 0 & T G_{2} & -2 T
\end{array}\right]
$$

$$
\begin{aligned}
& \beta_{1}=\dot{\xi}(t)^{\prime} M_{1}\left(-\dot{\xi}(t)+\mathcal{A}_{a} \xi(t)+\mathcal{A}_{b} z(t)+\left(\mathcal{A}_{c}-\mathcal{W} E_{c}\right) \psi\left(v_{c}(t)\right)\right) \\
& \beta_{2}=\xi(t)^{\prime} M_{2}\left(-\dot{\xi}(t)+\mathcal{A}_{a} \xi(t)+\mathcal{A}_{b} z(t)+\left(\mathcal{A}_{c}-\mathcal{W} E_{c}\right) \psi\left(v_{c}(t)\right)\right) \\
& \beta_{3}=z(t)^{\prime} M_{3}\left(\Omega_{a} \xi(t)+\Omega_{b} z(t)+\Omega_{c} \psi\left(v_{c}(t)\right)\right) \\
& \beta_{4}=\rho(t)^{\prime} M_{4}\left(\Xi_{1} \xi(t)+\Xi_{2} \rho(t)\right)
\end{aligned}
$$

In view of (6) and (7), it follows that the equations

$$
0=\beta_{1}+\beta_{1}^{\prime}, 0=\beta_{2}+\beta_{2}^{\prime}, 0=\beta_{3}+\beta_{3}^{\prime}, 0=\beta_{4}+\beta_{4}^{\prime}
$$

are satisfied, for any matrices $M_{1} \in \mathbb{R}^{n_{\xi} \times n_{\xi}}, M_{2} \in \mathbb{R}^{n_{\xi} \times n_{\xi}}, M_{3} \in \mathbb{R}^{n_{z} \times n_{z}}$ and $M_{4} \in \mathbb{R}^{n_{\rho} \times n_{\rho}}$.

From (24), if

$$
\zeta^{\prime} \Lambda_{2} \zeta+\beta_{1}+\beta_{1}^{\prime}+\beta_{2}+\beta_{2}^{\prime}+\beta_{3}+\beta_{3}^{\prime}-\beta_{4}-\beta_{4}^{\prime}<0
$$

holds, then (22) is satisfied. Observe that we can rewrite (25) as follows

$$
\zeta^{\prime} \Lambda_{3}(\xi) \zeta<0
$$

where

$$
\Lambda_{3}(\xi)=\left[\begin{array}{ccccc}
-M_{1}-M_{1}^{\prime} & P-M_{2}^{\prime}+M_{1} \mathcal{A}_{a} & M_{1} \mathcal{A}_{b} & 0 & M_{1} \mathcal{A}_{c}-M_{1} \mathcal{W} E_{c} \\
\star & M_{2} \mathcal{A}_{a}+\mathcal{A}_{a}^{\prime} M_{2}^{\prime} & M_{2} \mathcal{A}_{b}+\Omega_{a}^{\prime} M_{3}^{\prime} & -\Xi_{1}^{\prime} M_{4}^{\prime} & M_{2} \mathcal{A}_{c}-M_{2} \mathcal{W} E_{c}+G_{1}^{\prime} T \\
\star & \star & M_{3} \Omega_{b}+\Omega_{b}^{\prime} M_{3}^{\prime} & 0 & M_{3} \Omega_{c} \\
\star & \star & \star & -M_{4} \Xi_{2}-\Xi_{2} M_{4}^{\prime} & G_{2}^{\prime} T \\
\star & \star & \star & \star & -2 T
\end{array}\right] .
$$

Let us assume that $M_{1}, M_{3}$ and $M_{4}$ are nonsingular and that $M_{2}=M_{2}^{\prime}>0$. Define now the following matrices $Q_{1}=M_{1}^{-1}, Q_{2}=M_{2}^{-1}, Q_{3}=M_{3}^{-1}, Q_{4}=M_{4}^{-1}, F=T^{-1}$ and

$$
\Pi_{0}=\text { blockdiag }\left\{Q_{1}, Q_{2}, Q_{3}, Q_{4}, F\right\}
$$

Pre- and post-multiplying the condition $\Lambda_{3}(\xi)<0$ by $\Pi_{0}$ and $\Pi_{0}^{\prime}$, we have

$$
\left[\begin{array}{ccccc}
-Q_{1}-Q_{1}^{\prime} & Q_{1} P Q_{2}-Q_{1}+\mathcal{A}_{a} Q_{2} & \mathcal{A}_{b} Q_{3}^{\prime} & 0 & \mathcal{A}_{c} F-\mathcal{W} E_{c} F \\
\star & \mathcal{A}_{a} Q_{2}+Q_{2} \mathcal{A}_{a}^{\prime} & \mathcal{A}_{b} Q_{3}^{\prime}+Q_{2} \Omega_{a}^{\prime} & -Q_{2} \Xi_{1}^{\prime} & \mathcal{A}_{c} F-\mathcal{W} E_{c} F+Q_{2} G_{1}^{\prime} \\
\star & \star & \Omega_{b} Q_{3}^{\prime}+Q_{3} \Omega_{b}^{\prime} & 0 & \Omega_{c} F \\
\star & \star & \star & -\Xi_{2} Q_{4}^{\prime}-Q_{4} \Xi_{2}^{\prime} & Q_{4} G_{2}^{\prime} \\
\star & \star & \star & \star & -2 F
\end{array}\right]<0
$$


Observe that the above inequality is not an LMI due to the term $Q_{1} P Q_{2}$. However, in this case, we can consider $P=M_{2}$ and it follows that $P Q_{2}=I_{n_{\xi}}$. Besides, for the terms $Q_{2} G^{\prime}$ and $E_{c} F$, we consider the following change of variables: $V_{1}^{\prime}=Q_{2} G_{1}^{\prime}, V_{2}^{\prime}=Q_{4} G_{2}^{\prime}$ and $E_{F}=E_{c} F$. In this case, if

$$
\Lambda_{4}(\xi)<0
$$

with

$$
\Lambda_{4}(\xi)=\left[\begin{array}{ccccc}
-Q_{1}-Q_{1}^{\prime} & \mathcal{A}_{a} Q_{2} & \mathcal{A}_{b} Q_{3}^{\prime} & 0 & \mathcal{A}_{c} F-\mathcal{W} E_{F} \\
\star & \mathcal{A}_{a} Q_{2}+Q_{2} \mathcal{A}_{a}^{\prime} & \mathcal{A}_{b} Q_{3}^{\prime}+Q_{2} \Omega_{a}^{\prime} & -Q_{2} \Xi_{1}^{\prime} & \mathcal{A}_{c} F-\mathcal{W} E_{F}+V_{1}^{\prime} \\
\star & \star & \Omega_{b} Q_{3}^{\prime}+Q_{3} \Omega_{b}^{\prime} & 0 & \Omega_{c} F \\
\star & \star & \star & -\Xi_{2} Q_{4}^{\prime}-Q_{4} \Xi_{2}^{\prime} & V_{2}^{\prime} \\
\star & \star & \star & \star & -2 F
\end{array}\right],
$$

holds, then (22) is satisfied.

In light of the above, we state the following result.

Theorem 1 Consider system (5) satisfying A1-A2 and its DAR representation (6)-(7) satisfying A3. If there exist constant matrices $Q_{1}, Q_{2}=Q_{2}^{\prime}>0, Q_{3}, Q_{4}, E_{F}, V_{1}$ and $V_{2}$ of appropriate dimensions and a positive diagonal matrix $F$, satisfying the following matrix inequalities for all $\xi \in \mathcal{V}\left(\mathcal{B}_{\xi}\right)$ :

$$
\begin{gathered}
\Lambda_{4}(\xi)<0, \\
{\left[\begin{array}{cc}
Q_{2} & Q_{2} q_{r} \\
q_{r}^{\prime} Q_{2} & 1
\end{array}\right]>0, r=1, \ldots, n_{e}} \\
\left.\star \begin{array}{ccc}
Q_{2} & Q_{2} \Xi_{1}^{\prime} & Q_{2} K_{1(i)}^{\prime}-V_{1(i)}^{\prime} \\
\star & \Xi_{2} Q_{4}^{\prime}+Q_{4} \Xi_{2}^{\prime} & Q_{4} K_{2(i)}^{\prime}-V_{2(i)}^{\prime} \\
\star & \star & u_{0(i)}^{2}
\end{array}\right]>0, i=1, \ldots, n_{v}
\end{gathered}
$$

then the anti-windup gain $E_{c}=E_{F} F^{-1}$ is such that for all $\xi(0) \in \mathcal{R}$, with $P=Q_{2}^{-1}$, the respective closed-loop trajectories $\xi(t)$ belong to $\mathcal{R}$, and approach the origin as $t \rightarrow \infty$, where $\mathcal{R}$ is as given in (17).

Proof. First recall that matrices $\mathcal{A}_{a}, \mathcal{A}_{b}, \mathcal{A}_{c}, \Omega_{a}, \Omega_{b}, \Omega_{c}, K_{1}, K_{2}, \Xi_{1}$ and $\Xi_{2}$ are affine in $\xi$. If the inequalities (29)-(31) are feasible for each $\xi \in \mathcal{V}\left(\mathcal{B}_{\xi}\right)$, then they are also satisfied for all $\xi \in \mathcal{B}_{\xi}$ from similar convexity arguments of parameter-dependent LMIs [36].

Note that, since $\Omega_{b}$ and $\Xi_{2}$ are supposed to be nonsingular (from $\mathbf{A 3}$ ), the terms $\Omega_{b} Q_{3}^{\prime}+Q_{3} \Omega_{b}^{\prime}<0$ (from (29)) and $\Xi_{2} Q_{4}^{\prime}+Q_{4} \Xi_{2}>0$ (from (31)) ensure that $Q_{3}$ and $Q_{4}$ are nonsingular. Moreover, since (from (29)) $Q_{1}+Q_{1}^{\prime}>0$ and (by hypothesis) $Q_{2}>0$, it follows that matrix $\Pi_{0}$ defined in (27) is invertible. Hence if $\Lambda_{4}(\xi)<0$, it follows that $\Lambda_{3}(\xi)<0$. Hence, in view of $(24)$ we conclude that $(21)$ holds with $Q_{2}^{-1}=P>0$. Hence, if $\mathcal{R} \subset \mathcal{S} \cap \mathcal{B}_{\xi}$ and considering $V(\xi)=\xi^{\prime} P \xi$, it follows that $\dot{V}(\xi)<0, \forall \xi \in \mathcal{R}$, which ensures that for all $\xi(0) \in \mathcal{R}$ the result closed-loop trajectories $\xi(t)$ belong to $\mathcal{R}$ and approach the origin as $t \rightarrow \infty$. 
On the other hand, pre- and post-multiplying (30) by $\Pi_{1}=\operatorname{blockdiag}\left\{Q_{2}^{-1}, 1\right\}$ and $\Pi_{1}^{\prime}$, respectively, we obtain:

$$
\left[\begin{array}{cc}
P & q_{r} \\
q_{r}^{\prime} & 1
\end{array}\right] \geq 0, \quad \forall r=1, \ldots, n_{e} .
$$

which ensures that $\mathcal{R}$ is included in the region $\mathcal{B}_{\xi}$ defined in (8) [40].

Moreover, pre- and post-multiplying (31) by $\Pi_{2}=\operatorname{blockdiag}\left\{Q_{2}^{-1}, Q_{4}^{-1}, 1\right\}$ and $\Pi_{2}^{\prime}$, respectively, we obtain:

$$
\left[\begin{array}{ccc}
P & \Xi_{1}^{\prime} M_{4}^{\prime} & K_{1(i)}^{\prime}-G_{1(i)}^{\prime} \\
\star & M_{4} \Xi_{2}+\Xi_{2}^{\prime} M_{4}^{\prime} & K_{2(i)}^{\prime}-G_{2(i)}^{\prime} \\
\star & \star & u_{0(i)}{ }^{2}
\end{array}\right] \geq 0, \forall i=1, \ldots, n_{v} .
$$

Applying now the Schur's complement to (33) and in the sequel pre- and post-multiplying the obtained matrix inequality respectively by the vector $\left[\begin{array}{ll}\xi^{\prime} & \rho^{\prime}\end{array}\right]$ and its transpose, it follows that:

$$
\frac{\left(\left(K_{1(i)}-G_{1(i)}\right) \xi+\left(K_{2(i)}-G_{2(i)}\right) \rho\right)^{2}}{u_{0(i)}{ }^{2}} \leq \xi^{\prime} P \xi+2 \rho^{\prime} M_{4}\left(\Xi_{1} \xi+\Xi_{2} \rho\right), \quad \forall i=1, \ldots, n_{v}
$$

Hence, taking into account that $\Xi_{1} \xi+\Xi_{2} \rho=0$ and if $\xi^{\prime} P \xi \leq 1$, it follows that:

$$
\left|\left(K_{1(i)}-G_{1(i)}\right) \xi+\left(K_{2(i)}-G_{2(i)}\right) \rho\right| \leq u_{0(i)}, \quad \forall i=1, \ldots, n_{v}
$$

which ensures that $\mathcal{R} \subset \mathcal{S}$.

Hence we conclude that (30) and (31) ensure that $\mathcal{R} \subset \mathcal{B}_{\xi} \cap \mathcal{S}$, which concludes the proof.

Remark 2 In the particular case where $v_{c}$ depends linearly or bilinearly (quadratically) on $x$ and $\eta$, it follows that $\rho=0$ in (7) and thus we can consider $K_{2}=0, G_{2}=0, \Xi_{1}=0$ and $\Xi_{2}=0$.

In this case, relation (30) should be replaced by

$$
\left[\begin{array}{cc}
Q_{2} & Q_{2} K_{1(i)}^{\prime}-V_{1(i)}^{\prime} \\
\star & u_{0(i)}^{2}
\end{array}\right] \geq 0, \forall i=1, \ldots, n_{v} .
$$

Moreover, the $4^{\text {th }}$ line and the $4^{\text {th }}$ column in (29) should be eliminated.

\subsection{Optimization Problems}

The result given in Theorem 1 can be applied for computing an anti-windup gain in order to attempt a maximization of the region of attraction of the closed-loop system. In fact, this goal is implicitly achieved if we maximize the region $\mathcal{R}$ associated to the gain to be computed.

To this end, since $\mathcal{R}$ is an ellipsoidal domain, the following optimization problem can be considered:

$$
\min \operatorname{trace}(H):\left[\begin{array}{cc}
H & I \\
I & Q_{2}
\end{array}\right]>0,(29),(30),(31), \forall \xi \in \mathcal{V}\left(\mathcal{B}_{\xi}\right)
$$


Note that the minimization of $\operatorname{trace}(H)$ is a criterion that leads to the minimization of $\operatorname{trace}\left(Q_{2}^{-1}\right)=$ $\operatorname{trace}(P)$ an thus an implicit maximization of the size of $\mathcal{R}$. Other classical size criteria of ellipsoidal sets such as volume maximization, minor axis maximization and the maximization in certain directions (see, e.g., Chapter 2 in [37] and references therein) can also be applied. It should be noticed that (29), (30) and (31) are LMIs for $\xi \in \mathcal{V}\left(\mathcal{B}_{\xi}\right)$. Hence, problem (35) is convex and can be solved by standard LMI solvers.

Remark 3 The region $\mathcal{B}_{\xi}$ corresponds to a region where the feasibility of the state dependent LMIs of Theorem 1 should be verified. It is a priori fixed by the designer. In practice, it can be chosen as an hyperrectangle, which allows a straightforward description as (8) and the vertices characterization. Of course, the assumption regarding the existence and uniqueness of the solutions in $\mathcal{B}_{\xi}$ must be respected.

On the other hand, the size of the estimate $\mathcal{R}$ is highly dependent on the size of the polytope $\mathcal{B}_{\xi}$. In consequence, if $\mathcal{B}_{\xi}$ is small then the estimate $\mathcal{R}$, which is contained in $\mathcal{B}_{\xi}$, can be conservative. The combined maximization of $\mathcal{R}$ and $\mathcal{B}_{\xi}$ is a nonconvex problem, which is hard to be solved. A simple solution is to parameterize $\mathcal{B}_{\xi}$ as a hypercube, i.e.,

$$
\mathcal{B}_{\xi}=\left\{\xi:\left|\xi_{(i)}\right| \leq \alpha, i=1, \ldots, n_{\xi}\right\}
$$

and then starting from a sufficiently small $\alpha$ we can increase the value of $\alpha$ until the LMI conditions of Theorem 1 are not feasible. Alternatively, we may apply an iterative procedure consisting of two optimization steps. More precisely, if we parameterize $\mathcal{B}_{\xi}$ as below:

$$
\mathcal{B}_{\xi}:=\left\{\xi:\left|\xi_{(i)}\right| \leq \alpha_{i}, i=1, \ldots, n_{\xi}\right\}
$$

we solve (35) for given (sufficiently small) $\alpha_{1}, \ldots, \alpha_{n_{\xi}}$. Then, for given matrices $Q_{2}, Q_{3}, Q_{4}$ and $F$, we maximize $\mathcal{B}_{\xi}$ over $\alpha_{1}, \ldots, \alpha_{n_{\xi}}$ and free matrices $Q_{1}, E_{F}, V_{1}$ and $V_{2}$. Hence, we iterate between these two steps until there is no significant change in the size of $\mathcal{R}$.

Remark 4 The computational effort (number of decision variables and number of LMIs) for solving Theorem 1 or the optimization problem in (35) depends on the number of states $n$, the number of control states $n_{c}$, the number of control inputs $n_{v}$, the size $n_{z}$ of the auxiliary vector $z(t)$, the size $n_{\rho}$ of the auxiliary vector $\rho(t)$ and the polytope geometry (number of vertices and edges of $\mathcal{B}_{\xi}$ ). For a hyper-rectangle $\mathcal{B}_{\xi}$, the number of LMIs is given by $n_{L M I}=2^{n_{\xi}}\left(1+2 n_{\xi}+n_{v}\right)$ and the number of decision variables is $n_{d v}=n_{\xi}^{2}+n_{\xi}\left(n_{\xi}+1\right) / 2+n_{\rho}^{2}+n_{z}^{2}+n_{v}\left(n_{\xi}+n_{\rho}+n_{c}+1\right)$, where $n_{\xi}=n+n_{c}$.

\section{Numerical Examples}

Example 1 Consider the nonlinear closed-loop system treated in [24]:

$$
\begin{aligned}
\dot{x}(t) & =\left(x^{2}(t)-1\right) x(t)+\operatorname{sat}\left(v_{c}(t)\right) \\
y(t) & =x(t),
\end{aligned}
$$


with $u_{0}=1$, and the controller

$$
\begin{aligned}
\dot{\eta}(t) & =-x(t) \\
v_{c}(t) & =\eta(t)-2 y(t) .
\end{aligned}
$$

Considering the DAR given in (6) with $z=x^{2}$, we get for (36)-(37) the following:

$$
\begin{gathered}
\mathcal{A}_{a}=\left[\begin{array}{ll}
-3 & 1 \\
-1 & 0
\end{array}\right], \mathcal{A}_{b}=\left[\begin{array}{l}
x \\
0
\end{array}\right], \mathcal{A}_{c}=\left[\begin{array}{c}
-1 \\
0
\end{array}\right], \\
\Omega_{a}=\left[\begin{array}{ll}
x & 0
\end{array}\right], \Omega_{b}=-1, \Omega_{c}=0, K_{1}=\left[\begin{array}{ll}
-2 & 1
\end{array}\right] .
\end{gathered}
$$

Note that in this case (see Remark 2), $\rho=0$ and thus $K_{2}=0, \Xi_{1}=0$ and $\Xi_{2}=0$.

Consider $\mathcal{B}_{\xi}:=\left\{\xi \in \mathbb{R}^{2}:\left|\xi_{1}\right| \leq \alpha_{1},\left|\xi_{2}\right| \leq \alpha_{2}\right\}$, where $\alpha_{1}=1.3$ and $\alpha_{2}=2.4$. Based on optimization problem stated in (35), we have first determined the estimate $\mathcal{R}_{1}$ of the region of attraction of the closed-loop system without anti-windup compensation, i.e. forcing $E_{c}=0$. Figure 1 shows the obtained estimate $\mathcal{R}_{1}$ and the actual region of attraction, which is clearly bounded by a limit cycle. In this case the matrix $P$ is given by:

$$
P=\left[\begin{array}{cc}
0.9826 & -0.2750 \\
-0.2750 & 0.3211
\end{array}\right]
$$

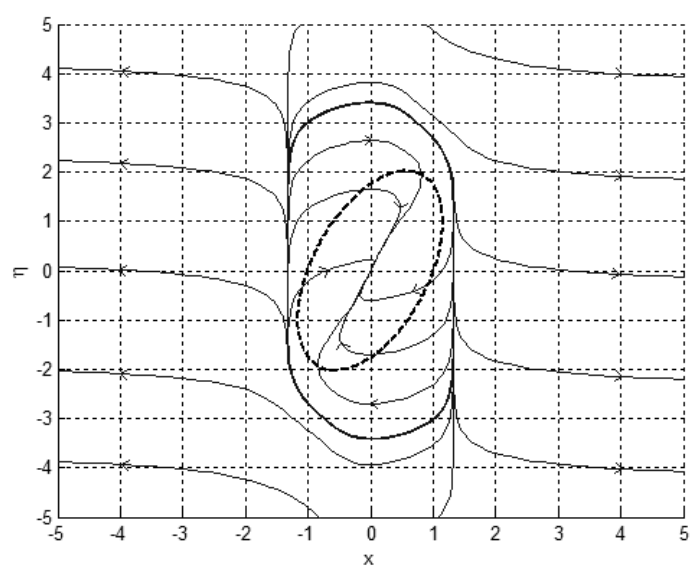

Figure 1: Example 1 - Estimate of the region of attraction $\mathcal{R}_{1}$ without anti-windup.

Applying now the optimization problem (35) and considering $E_{c} \neq 0$, we obtain:

$$
P=\left[\begin{array}{cc}
0.8514 & -0.2547 \\
-0.2547 & 0.2498
\end{array}\right] \text { and } E_{c}=5.2464
$$

Figure 2 shows the new estimate of the region of attraction for the above value of $E_{c}$, which is denoted by $\mathcal{R}_{2}$. The resulting region of attraction in this case can also be observed in the figure. Note that the introduction of the anti-windup compensation makes the limit cycle disappear and now the region of attraction is clearly bigger and unbounded in some directions. 


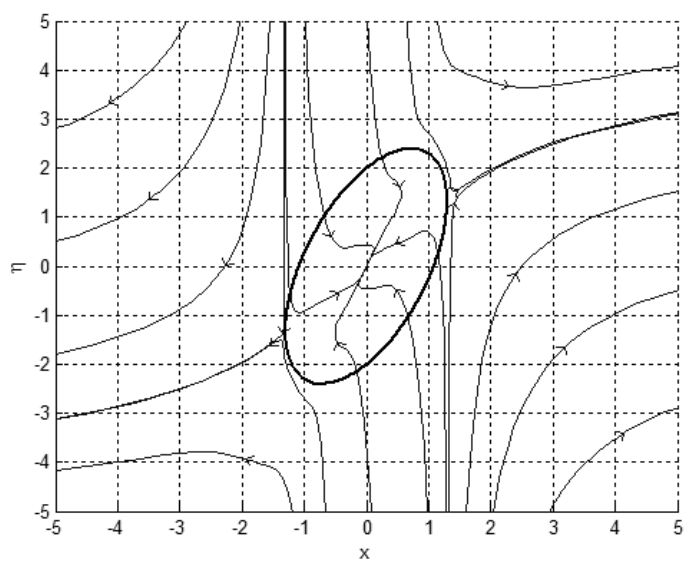

Figure 2: Example 1 - Estimate of the region of attraction $\mathcal{R}_{2}$ with anti-windup.

For comparison purposes, we present both estimates of the region of attraction in Figure 3, where $\mathcal{R}_{1}$ is in dashed line and $\mathcal{R}_{2}$ is in solid line. The region where the control does not saturate is denoted by $\mathcal{R}_{n s}$.

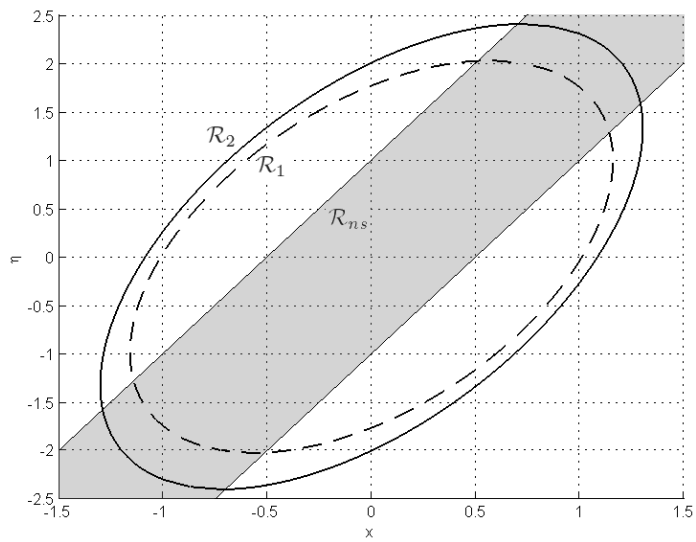

Figure 3: Example 1 - Comparison of $\mathcal{R}_{1}$ and $\mathcal{R}_{2}$.

Considering now the initial condition $\xi(0)=\left[\begin{array}{ll}-0.6 & 1.16\end{array}\right]^{\prime}$, Figure 4 shows the time evolution of the output (y) and the control signal $\left(s a t\left(v_{c}\right)\right)$ in 3 cases, namely: present approach, approach from [24] and without the anti-windup strategy. Note that, with anti-windup strategy the control signal remains less time saturated and the transient performance is improved. Although the performance optimization is not the focus neither in [24] nor in the present work, we can note that a better performance is obtained with the anti-windup computed from the present approach.

It should also be pointed out that conditions in [24] are BMIs and an iterative algorithm is proposed to obtain the estimate of the region of attraction, which leads to a greater computational burden. Moreover, this algorithm is strongly dependent on the initialization and in general only suboptimal solutions can be achieved. 

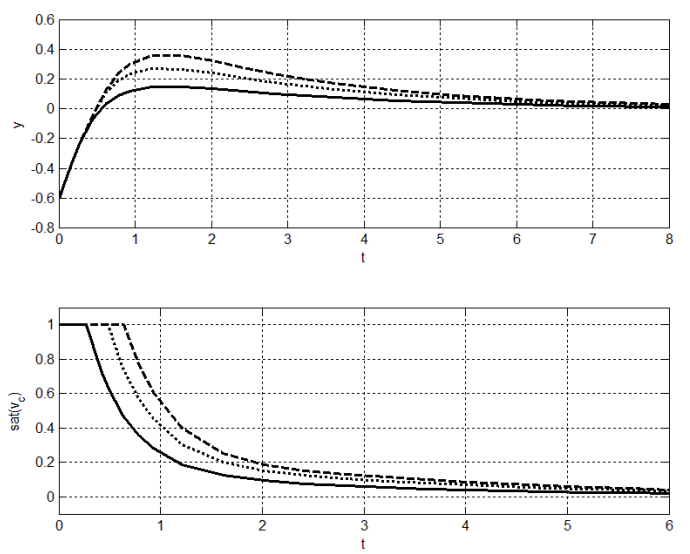

Figure 4: Example 1 - Time evolution of the output $y$ and the control signal with the present approach (solid), the approach in [24] (dotted) and without AW (dashed).

Example 2 Consider the closed-loop system borrowed from [22]:

$$
\begin{aligned}
\dot{x}_{1}(t) & =x_{1}(t)+2 x_{1}(t)^{2}+x_{1}(t) x_{2}(t)+\operatorname{sat}\left(v_{c}(t)\right) \\
\dot{x}_{2}(t) & =x_{1}(t) \\
y(t) & =x_{1}(t)+0.5 x_{2}(t)
\end{aligned}
$$

with $u_{0}=0.6$ and the NDI controller

$$
\begin{aligned}
& \dot{\eta_{1}}(t)=x_{1}(t)+0.5 x_{2}(t)-1.480 \eta_{1}(t)+2.450 \eta_{2}(t) \\
& \dot{\eta_{2}}(t)=2.450 \eta_{1}(t) \\
& v_{c}(t)=-2 x_{1}(t)^{2}-x_{1}(t) x_{2}(t)-4.4 x_{1}(t)-2.2 x_{2}(t)-15.148 \eta_{1}(t)-21.449 \eta_{2}(t) .
\end{aligned}
$$

Considering the DAR given in (6), we get for (38)-(39) the following:

$$
\mathcal{A}_{a}=\left[\begin{array}{cccc}
-3.4 & -2.2 & -15.148 & -21.449 \\
1 & 0 & 0 & 0 \\
1 & 0.5 & -1.480 & 2.450 \\
0 & 0 & 2.450 & 0
\end{array}\right], \mathcal{A}_{c}=\left[\begin{array}{c}
-1 \\
0 \\
0 \\
0
\end{array}\right],
$$

and

$$
K_{1}=\left[\begin{array}{llll}
-4.4-2 x_{1} & -2.2-x_{1} & -15.148 & -21.449
\end{array}\right] .
$$

Since the open-loop system and the output of the controller are quadratic in $x$ and $\eta$, in this case $\mathcal{A}_{b}, \Omega_{a}$, $\Omega_{b}, \Omega_{c}, K_{2}, \Xi_{1}$ and $\Xi_{2}$ are equal to zero matrices of appropriate dimensions.

Consider $\mathcal{B}_{\xi}:=\left\{\xi \in \mathbb{R}^{4}:\left|\xi_{1}\right| \leq \alpha_{1},\left|\xi_{2}\right| \leq \alpha_{2},\left|\xi_{3}\right| \leq \alpha_{3},\left|\xi_{4}\right| \leq \alpha_{4},\right\}$, where $\alpha_{1}=0.35$ and $\alpha_{2}=\alpha_{3}=$ $\alpha_{4}=0.6$. Based on optimization problem stated in (35), we have first determined the estimate $\mathcal{R}_{1}$ of the region of attraction of the closed-loop system without anti-windup compensation, i.e. forcing $E_{c}=0$. In this 
case the matrix $P$ is given by:

$$
P=\left[\begin{array}{cccc}
56.3 & 23.3 & 113.2 & 154.1 \\
23.3 & 20.8 & 76.7 & 105.4 \\
113.2 & 76.7 & 611.2 & 834.3 \\
154.1 & 105.4 & 834.3 & 1155.4
\end{array}\right]
$$

with $\operatorname{trace}(P)=1843.71$.

Applying now the optimization problem (35) and considering $E_{c} \neq 0$, we obtain a region $\mathcal{R}_{2}$, defined from

$$
P=\left[\begin{array}{cccc}
20.0139 & 8.4749 & 36.9308 & 49.1887 \\
8.4749 & 6.6146 & 30.3210 & 40.6876 \\
36.9308 & 30.3210 & 220.9461 & 301.6380 \\
49.1887 & 40.6876 & 301.6380 & 412.5945
\end{array}\right]
$$

with $\operatorname{trace}(P)=660.17$ and $E_{c}=\left[\begin{array}{c}-5.0000 \\ 3.4285\end{array}\right]$. Observe that the trace of $P$ is significantly reduced by using the anti-windup technique, which implicitly means that the region of attraction is enlarged with the anti-windup compensation.

Consider now the initial condition $\xi(0)=\left[\begin{array}{llll}0.2235 & 0 & 0 & 0\end{array}\right]^{\prime}$. It should be noticed that $\xi(0) \in \mathcal{R}_{2}$, but $\xi(0)$ does not belong to $\mathcal{R}_{1}$. Figure 5 shows the response of the output $y$ and the control signal sat $\left(v_{c}\right)$ for this initial condition considering the cases with (solid line) and without anti-windup strategy (dashed line). It can be seen that without anti-windup compensation the closed-loop trajectory diverges.
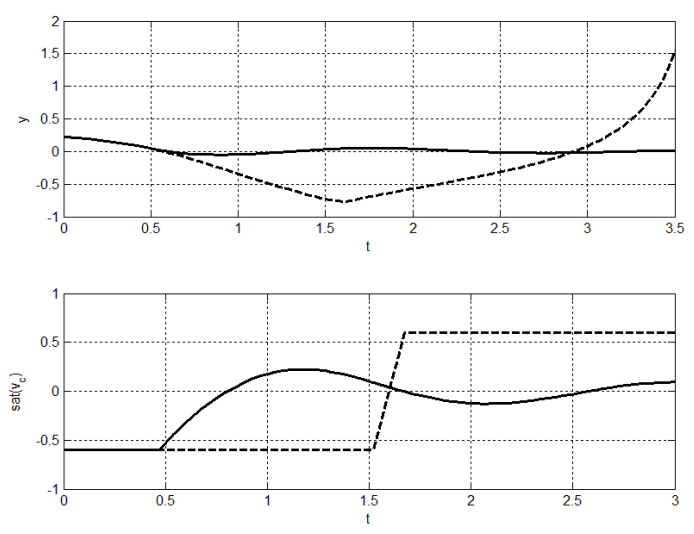

Figure 5: Example 2 - Time evolution of the output $y$ and the control signal $v_{c}$ with AW (solid) and without AW (dashed).

Let us now discuss our results with respect to those proposed in [22], which concerns the design of $n$ order dynamic anti-windup controllers (with an additional state $x_{a}$ ), and aims at providing an estimate of the 
domain of attraction in the subspace of the plant state $x$. In the current paper, we are concerned with a static anti-windup, and we consider the whole augmented state for the estimation of the region of attraction. Then, in order to compare both approaches, we plot in Figure 6 the cut of the estimates of the region of attraction in the subespace defined by the state $x$ (i.e considering $x_{c}=0$ and, for the dynamic anti-windup case, also $\left.x_{a}=0\right)$. Although the Lyapunov function used in [22] is more complex (i.e. it presents bi-quadratic terms in $x, x_{c}$ and $\left.x_{a}\right)$, it can be shown that the cut of the obtained estimate of the region of attraction is included in the ellipoidal set plotted in dashed line. It can be observed that the region associated to our approach clearly includes the dashed ellipsoid, which shows that the set of plant initial conditions for which the stability is guaranteed ensured is larger than the one provided by [22].

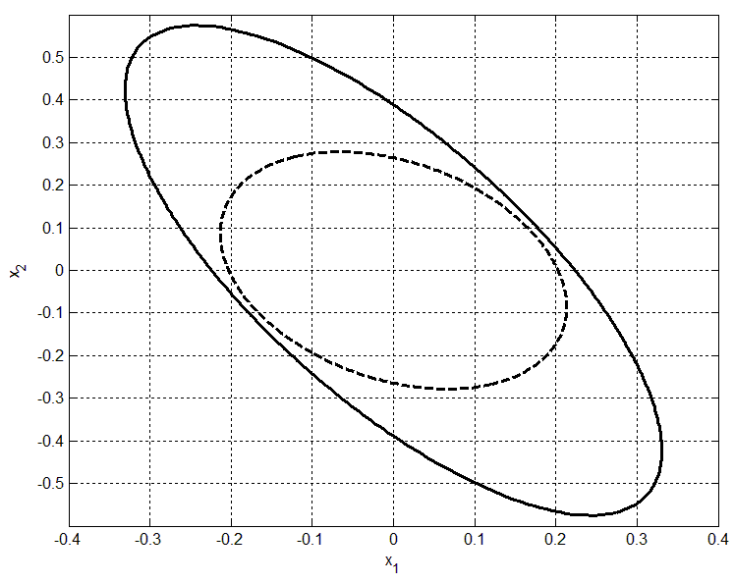

Figure 6: Example 2 - Cut of the stability regions on the subspace defined by the plant states: present approach (solid) and approach from [22] (dashed).

Although the performance optimization is not the focus neither in [22] nor in our paper, in Figure 7 a comparison between the time-responses considering an initial condition $\xi(0)=\left[\begin{array}{lllll}0.2235 & 0 & 0 & 0\end{array}\right]^{\prime}$ is depicted. For the dynamic anti-windup compensator computed in [22] we considered $x_{a}(0)=0$. We can note that the response with our approach is slightly better. Moreover, it should be pointed out that in our case the anti-windup structure (i.e. a simple gain) is much simpler than the $n$-order dynamic nonlinear structure proposed in [22].

Example 3 Consider the following multivariable nonlinear system and assume that the inputs are subject to saturation, with $u_{0}=\left[\begin{array}{ll}1 & 1\end{array}\right]^{\prime}$ :

$$
\begin{aligned}
& \dot{x}_{1}(t)=\left(x_{1}(t)+2\right) x_{1}^{2}(t)+10 x_{2}(t)+10 \operatorname{sat}\left(v_{c 2}(t)\right) \\
& \dot{x}_{2}(t)=-100 x_{1}(t)-30 x_{2}(t)+10 \operatorname{sat}\left(v_{c 1}(t)\right) \\
& y_{1}(t)=20 x_{1}(t) \\
& y_{2}(t)=20 x_{2}(t) .
\end{aligned}
$$



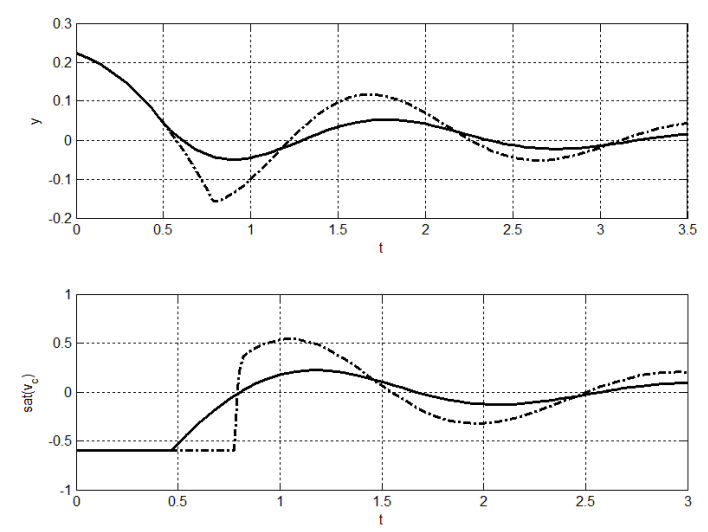

Figure 7: Example 2 - Time evolution of the output $y$ : present approach (solid) and approach from [22] (dashed).

From the linearized model of system (40), we designed a locally stabilizing multivariable proportionalintegral (PI) controller given by:

$$
\begin{aligned}
\dot{\eta}(t) & =A_{\eta} x(t)+B_{\eta} y(t) \\
v_{c}(t) & =C_{\eta} \eta(t)+D_{\eta} \eta(t),
\end{aligned}
$$

where $\eta(t)=\left[\begin{array}{ll}\eta_{1}(t)^{\prime} & \eta_{2}(t)^{\prime}\end{array}\right]^{\prime}, v_{c}(t)=\left[\begin{array}{ll}v_{c_{1}}(t)^{\prime} & v_{c_{2}}(t)^{\prime}\end{array}\right]^{\prime}$,

$$
A_{\eta}=0_{2}, \quad B_{\eta}=I_{2}, \quad C_{\eta}=\left[\begin{array}{cc}
-0.4686 & -0.3703 \\
-0.2959 & 0.4149
\end{array}\right], D_{\eta}=\left[\begin{array}{cc}
0.0728 & 0.0516 \\
-0.0227 & 0.0176
\end{array}\right] \text {. }
$$

Considering $z=x_{1}{ }^{2}$, the following DAR for (40)-(41) can be obtained:

$$
\begin{gathered}
\mathcal{A}_{a}=\left[\begin{array}{cccc}
-4.5400 & 13.5200 & -2.9590 & 4.1490 \\
-85.4400 & -19.6800 & -4.6860 & -3.7030 \\
20.0000 & 0 & 0 & 0 \\
0 & 20.0000 & 0 & 0
\end{array}\right], \mathcal{A}_{b}=\left[\begin{array}{c}
x_{1}+2 \\
0 \\
0 \\
0
\end{array}\right], \mathcal{A}_{c}=\left[\begin{array}{c}
-10 \\
-10 \\
0 \\
0
\end{array}\right], \\
\Omega_{a}=\left[\begin{array}{llll}
x_{1} & 0 & 0 & 0
\end{array}\right], \Omega_{b}=-1 \\
\text { and } K_{1}=\left[\begin{array}{cccc}
1.4560 & 1.0320 & -0.4686 & -0.3703 \\
-0.4540 & 0.3520 & -0.2959 & 0.4149
\end{array}\right],
\end{gathered}
$$

In this case, $\rho=0$ and thus $K_{2}=0, \Xi_{1}=0$ and $\Xi_{2}=0$. Moreover, since the matrices of the DAR depend only on the state $x_{1}$, we can consider $\mathcal{B}_{\xi}:=\left\{\xi \in \mathbb{R}^{5}:\left|x_{1}\right| \leq \alpha_{1}\right\}$. For $\alpha_{1}=3.0$, Table 1 shows the results of the optimization problem (35), considering the case without and with anti-windup compensation. From the matrices $P$ and the respective traces, we can conclude that the estimate $\mathcal{R}$ of the region of attraction is significantly enlarged with the application of the anti-windup compensation. 


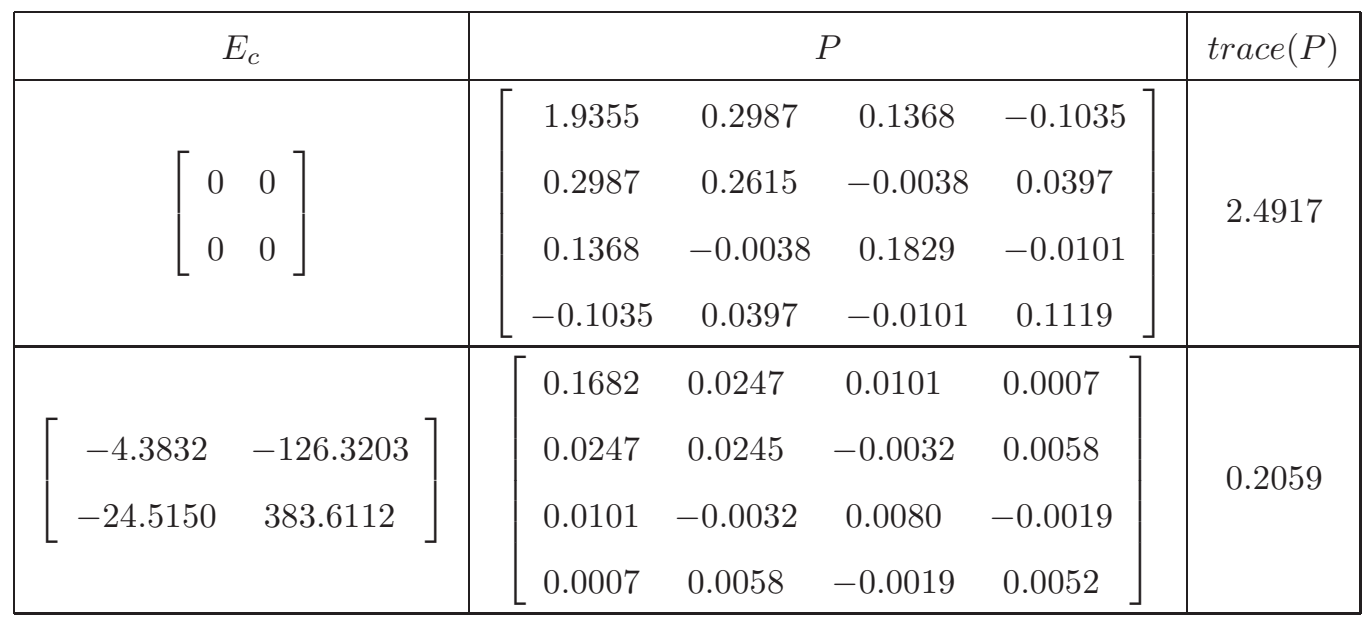

Table 1: Example 3 - Results of the optimization problem for $E_{c}=0$ and $E_{c} \neq 0$.

Figure 8 shows the time evolution of the outputs and the control signals obtained from the initial condition $\xi(0)=\left[\begin{array}{llll}-0.1548 & -1.0109 & -3.2397 & 13.0463\end{array}\right]^{\prime}$ considering the cases with and without anti-windup strategy. It should be noticed that $\xi(0)$ belongs to the region $\mathcal{R}$ obtained with anti-windup, but it does not belong to the one obtained without ant-windup. We can observe that with the anti-windup compensation (solid-line), the control remains less time in saturation and the convergence of the closed-loop trajectories to the origin is significantly faster.
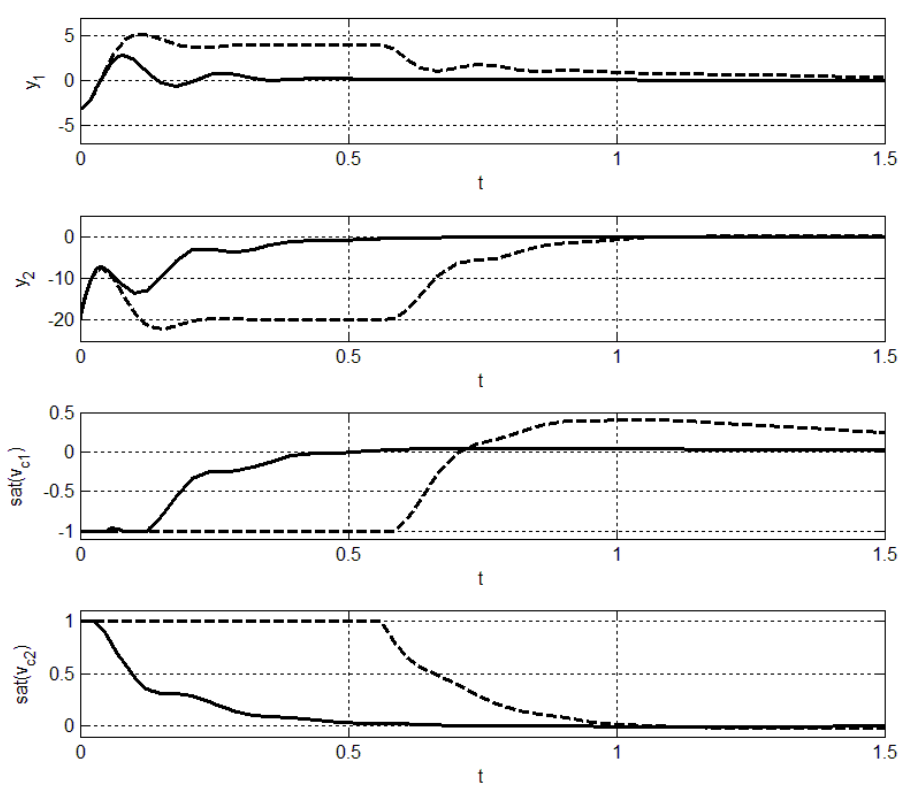

Figure 8: Example 3 - Time evolution of the outputs $y$ and the control signals $v_{c}$ with AW (solid) and without AW (dashed). 


\section{Concluding Remarks}

This paper has proposed an approach to compute anti-windup gains for a class of multivariable nonlinear systems subject to actuator saturation. The proposed design conditions relies on a differential algebraic representation of rational systems, which can model a broad class of nonlinear systems. To deal with the saturation, we have considered a modified version of the generalized sector bound condition. From these elements, an LMI-based method has been devised to compute anti-windup gains aiming at the maximization of the estimates of the region of attraction of the closed-loop system. Several numerical examples have illustrated the application of the proposed approach and the effective improvement on the size of the region of attraction of the closed-loop system achieved by means of an appropriate anti-windup compensation.

\section{Acknowledgements}

J.M. Gomes da Silva Jr. was partially supported by CAPES (BEX 3594-10-6) and CNPq (grant 306210/20096/PQ). M. Z. Oliveira received a CAPES PhD scholarship. D. Coutinho was partially supported by CNPq (grants 477390/2010-2 and 302136/2011-8/PQ).

\section{References}

[1] S. Tarbouriech and M. Turner, "Anti-windup Design: An Overview of Some Recent Advances and Open Problems," IET Control Theory \& Applications, vol. 3, no. 1, pp. 1-19, 2009.

[2] A. R. Teel and N. Kapoor, "The $\mathcal{L}_{2}$ Anti-windup Problem: Its Definition and Solution," in Proceedings of the European Control Conference, Brussels, Belgium, 1997.

[3] S. Galeani, S. Tarbouriech, M. Turner, and L. Zaccarian, "A Tutorial on Modern Anti-windup Design," European Journal of Control, vol. 15, no. 3-4, pp. 418-440, 2009.

[4] L. Zaccarian and A. Teel, Modern Anti-windup Synthesis: Control Augmentation for Actuator Saturation. Princeton University Press, 2011.

[5] Y.-Y. Cao, Z. Lin, and D. G. Ward, "An Antiwindup Approach to Enlarging Domain of Attraction for Linear Systems Subject to Actuator Saturation," IEEE Transactions on Automatic Control, vol. 47, no. 1, pp. 140-145, 2002.

[6] J. M. Gomes da Silva Jr. and S. Tarbouriech, "Anti-windup Design with Guaranteed Regions of Stability: An LMI-based Approach," IEEE Transactions on Automatic Control, vol. 50, no. 1, pp. 106-111, 2005.

[7] E. Prempain, M. Turner, and I. Postlethwaite, "Coprime Factor Based Anti-windup Synthesis for Parameter-dependent Systems," System E Control Letters, vol. 58, no. 12, pp. 810-817, 2009. 
[8] F. Wu, K. M. Grigoriadis, and A. Packard, "Anti-windup Controller Design Using Linear Parametervarying Control Methods," International Journal of Control, vol. 73, no. 12, pp. 1104-1114, 2000.

[9] F. Wu and M. Soto, "Extended anti-windup control schemes for LTI and LFT systems with actuator saturations," Int. J. of Robust and Nonlinear Control, vol. 14, pp. 1255-1281, 2004.

[10] F. Morabito, A. Teel, and L. Zaccarian, "Nonlinear Anti-windup Applied to Euler-Lagrange Systems," IEEE Transactions on Robotics and Automation, vol. 20, no. 3, pp. 526-537, 2004.

[11] N. Kahveci, P. Ioannou, and M. Mirmirani, "A Robust Adaptive Control Design for Gliders Subject to Actuator Saturation Nonlinearities," in Proceedings of the American Control Conference, New York City, United States, 2007, pp. 492-497.

[12] G. Herrmann, P. P. Menon, M. C. Turner, D. G. Bates, and I. Postlethwaite, "Anti-windup Synthesis for Nonlinear Dynamic Inversion Control Schemes," International Journal of Robust and Nonlinear Control, vol. 20, pp. 1465-1482, 2010.

[13] T. A. Kendi and F. J. Doyle III, "An Anti-windup Scheme for Multivariable Nonlinear Systems," Journal of Process Control, vol. 7, no. 5, pp. 329-343, 1997.

[14] N. Kapoor and P. Daoutidis, "An Observer-based Anti-windup Scheme for Non-linear Systems with Input Constraints," International Journal of Control, vol. 72, no. 1, pp. 18-29, 1999.

[15] F. J. Doyle III, "An Anti-windup Input-output Linearization Scheme for SISO Systems," Journal of Process Control, vol. 9, no. 3, pp. 213-220, 1999.

[16] P. Menon, G. Herrmann, M. Turner, D. Bates, and I. Postlethwaite, "General Anti-windup Synthesis for Input Constrained Nonlinear Systems Controlled Using Nonlinear Dynamic Inversion," in Proceedings of the 45th IEEE Conference on Decision and Control, San Diego, United States, 2006, pp. 5435-5440.

[17] H. K. Khalil, Nonlinear Systems. Prentice Hall, 1996.

[18] A. Barreiro, J. Aracil, and D. Pagano, "Detection of Attraction Domains of Nonlinear Systems Using the Bifurcation Analysis and Lyapunov Functions," International Journal of Control, vol. 75, no. 5, pp. $314-327,2006$.

[19] D. F. Coutinho and J. M. Gomes da Silva Jr., "Computing Estimates of the Region of Attraction for Rational Control Systems with Saturating Actuators," IET Control Theory and Applications, vol. 4, no. 3, pp. 315-325, 2010.

[20] G. Chesi, "Estimating the domain of attraction for uncertain polynomial systems," Automatica, vol. 40, pp. 1981-1986, 2004. 
[21] , Domain of Attraction - Analysis and Control via SOS Programming. Springer, 2011.

[22] G. Valmorbida, S. Tarbouriech, M. Turner, and G. Garcia, "Anti-windup for NDI Quadratic Systems," in Proceedings of the 8th IFAC Symposium on Nonlinear Control Systems, vol. 1, Bologna, Italy, 2010, pp. $1175-1180$.

[23] P. Weston and I. Postlethwaite, "Analysis and Design of Linear Conditioning Schemes for Systems Containing Saturating Actuators," in Proceedings of the IFAC Nonlinear Control System Design Symposium, 1998.

[24] M. Z. Oliveira, J. M. Gomes da Silva Jr., D. F. Coutinho, and S. Tarbouriech, "Anti-windup Design for a Class of Nonlinear Control Systems," in Proceedings of 18th World Congress of the International Federation of Automatic Control, Milano, Italy, 2011.

[25] — - "Anti-windup Design for a Class of Multivariable Nonlinear Control Systems: an LMI-based Approach," in Proceedings of IEEE Conf. on Decision and Control, Orlando, USA, 2011.

[26] L.A. Aguirre and C. Letellier, "Modeling Nonlinear Dynamics and Chaos: A Review," Mathematical Problems in Engineering, vol. 2009, Article id 238960, 35 pages, 2009.

[27] J. Nemcova and J.H. van Schuppen, "Realization theory for rational systems: the existence of rational realizations," SIAM Journal on Control and Optimization, vol. 48, no. 4, pp. 2840-2856, 2009.

[28] L. El Ghaoui and G. Scorletti, "Control of Rational Systems Using Linear-Fractional Representations and LMIs," Automatica, vol. 32, no. 9, pp. 1273-1284, 1996.

[29] S. Dussy, "Multiobjective robust control toolbox for linear-matrix-inequality-based control," in Advances in Linear Matrix Inequality Methods in Control, L. El Ghaoui and S. Niculescu, Eds. SIAM, 2000, pp. 309-320.

[30] D.F. Coutinho, A. Trofino and M. Fu, "Guaranteed cost control of uncertain nonlinear systems via polynomial Lyapunov functions," IEEE Transactions on Automatic Control, vol. 47, no. 9, pp. 1575$1580,2002$.

[31] F. Wang and V. Balakrishnan, "Improved stability analysis and gain-scheduled controller synthesis for parameter-dependent systems," IEEE Transactions on Automatic Control, vol. 47, no. 5, pp. 720-734, 2002.

[32] D. F. Coutinho, A. S. Bazanella, A. Trofino, and A. S. Silva, "Stability Analysis and Control of a Class of Differential-Algebraic Nonlinear Systems," International Journal of Robust and Nonlinear Control, vol. 14, no. 16, pp. 1301-1326, 2004. 
[33] A. Papachristodoulou and S. Prajna, "Analysis of non-polynomial systems using the sum of squares decomposition," in Positive Polynomials in Control (Lecture Notes in Control and Information Sciences, Vol. 312), D. Henrion and A. Garulli, Eds. Springer-Verlag, 2005, pp. 23-43.

[34] D. F. Coutinho, M. Fu, A. Trofino, and P. Danes, " $\mathcal{L}_{2}$-Gain Analysis and Control of Uncertain Nonlinear Systems with Bounded Disturbance Inputs," International Journal of Robust and Nonlinear Control, vol. 18, pp. 88-110, 2008.

[35] B. Acikmese and M. Corless, "Stability analysis with quadratic Lyapunov functions: Some necessary and sufficient multiplier conditions," System 83 Control Letters, vol. 57, pp. 78-94, 2008.

[36] F. Amato, Robust Control of Linear Systems Subject to Uncertain Time-Varying Parameters. SpringerVerlag, 2006.

[37] S. Tarbouriech, G. Garcia, J. M. Gomes da Silva Jr. and I. Queinnec, Stability and Stabilization of Linear Systems with Saturating Actuators. Springer, 2011.

[38] H. Hindi and S. Boyd, "Analysis of Linear Systems with Saturation Using Convex Optimization," in Proceedings of the 37th IEEE Conference on Decision and Control, Tampa, United States, 1998, pp. 903-908.

[39] T. Kiyama and T. Iwasaki, "On the Use of Multi-loop Circle Criterion for Saturating Control Synthesis," in Proceedings of the American Control Conference, Chicago, United States, 2000, pp. 1225-1229.

[40] S. Boyd, L. El Ghaoui, E. Feron, and V. Balakrishnan, Linear Matrix Inequalities in Systems and Control Theory. SIAM books, 1994. 